\title{
Ambiguity Seeking as a Result of the Status Quo Bias
}

\author{
Mercè Roca ${ }^{1}$, Robin M. Hogarth ${ }^{2}$, A. John Maule ${ }^{3}$ \\ ${ }^{1}$ ESRC Postgraduate Researcher, Centre for Decision Research, Leeds University Business School, The University of \\ Leeds, Leeds, LS2 9JT, United Kingdom \\ ${ }^{2}$ ICREA Research Professor, Departament d'Economia i Empresa, Universitat Pompeu Fabra, Ramon Trias Fargas, \\ 08005, Barcelona, Spain \\ ${ }^{3}$ Professor, Centre for Decision Research, Leeds University Business School, The University of Leeds, Leeds, LS2 \\ 9JT, United Kingdom
}

Contact details: Mercè Roca, busmr@leeds.ac.uk, Telephone: (0044)07709557423. Leeds University Business School, Maurice Keyworth Building. The University of Leeds, Leeds, LS2 9JT, United Kingdom

* Funds for the experiments were provided by the Centre for Decision Research of Leeds University Business School. The authors are grateful for the comments received from Gaëlle Villejoubert, Albert Satorra and Peter Wakker. This paper was presented at SPUDM20 in Stockholm. 


\begin{abstract}
Several factors affect attitudes toward ambiguity. What happens, however, when people are asked to exchange an ambiguous alternative in their possession for an unambiguous one? We present three experiments in which individuals preferred to retain the former. This status quo bias emerged both within- and between-subjects, with and without incentives, with different outcome distributions, and with endowments determined by both the experimenter and the participants themselves. Findings emphasize the need to account for the frames of reference under which evaluations of probabilistic information take place as well as modifications that should be incorporated into descriptive models of decision making.
\end{abstract}

\title{
Keywords
}

Ambiguity, risk, status quo bias, decision making, uncertainty.

JEL code: C91, D81. 
The phenomenon of ambiguity aversion - or the preference for gambles with known as opposed to unknown probabilities - has been well documented in the literature on decision making in both psychology and economics (see, e.g., Ellsberg, 1961; Camerer \& Weber, 1992; Keren \& Gerritsen, 1999). Indeed, that the notion of ambiguity aversion is now well accepted in economics can be demonstrated by its use to explain, for example, certain phenomena in financial markets (Mukerji \& Tallon, 2001).

At the same time, however, situations have been identified where people might prefer ambiguous alternatives. Ellsberg (cited in Becker \& Brownson, 1964), suggested that people may prefer ambiguous alternatives when known probabilities are small (cf., Einhorn \& Hogarth, 1986, who also consider effects of losses and larger probabilities). In addition, Heath and Tversky (1991) demonstrated a so-called competence effect whereby people prefer ambiguous alternatives when they feel especially competent or knowledgeable about the source of uncertainty (see also Fox \& Tversky, 1995).

In experiments on ambiguity people are typically asked to choose between alternatives characterized by different types of uncertainty. Curiously, however, they are not asked to choose between something they already own and an alternative they could accept in exchange where both have uncertain outcomes. ${ }^{1}$ And yet, this latter form of choice is quite common in economic transactions. Consider, for example, choices between, say, holding onto bonds (stocks) or exchanging them for stocks (bonds). Other examples could include the exchange between something you have already purchased - such as a vacation package - for another alternative.

The purpose of the present paper is to investigate the effects of ambiguity in these kinds of situations. But first we note that neither classic economic reasoning nor descriptive theories of ambiguity distinguish between the two types of decision, i.e., choice between alternatives versus exchanging alternatives. On the other hand, many 
descriptive findings suggest that people "overvalue" what they currently own and that this can affect their willingness to exchange goods. This has been labeled the "status quo" bias (Samuelson \& Zeckhauser, 1988). A related line of research refers to the "endowment effect" or the fact that willingness-to-pay (WTP) prices for goods are typically much smaller than willingness-to-accept (WTA) prices if the goods are already in a person's possession, i.e., are part of their endowment (Thaler, 1980; Kahneman, Knetsch, \& Thaler, 1991). These findings are, of course, consistent with loss aversion in prospect theory that depends on the reference point used to describe a person's assets (Kahneman \& Tversky, 1979). However, one could also think of reference points in terms of levels of probability (Viscusi, Magat, \& Huber, 1987).

The specific question we ask centers on what happens when the forces that lead to ambiguity aversion are confronted by those of the endowment effect. In other words, will a person who owns an alternative with ambiguous outcomes exchange it for an alternative where the probabilities are known? Consider an example: When choosing between stocks and bonds, a person selects the bonds. However, had the person owned those same stocks, would he or she have exchanged them for the bonds?

This paper is organized as follows. We test the potential conflict between ambiguity aversion and the endowment effect in three experiments. In the first, we use an Ellsberg-like task and demonstrate that participants endowed with ambiguous gambles are reluctant to exchange these for their non-ambiguous counterparts. These results were achieved using a between-subjects experimental design and without proper financial incentives. In Experiment 2, therefore, our aim was to replicate these results using both a within-subjects experimental design and proper financial incentives. By using this design, we sought to provide participants with the opportunity to question their own behavior and, yet, we still observed ambiguity seeking consistent with the 
status quo bias. Experiment 1 and 2 both used abstract stimuli involving gambles. Thus in Experiment 3, we replicated Experiment 2 using a meaningful stock market context instead of gambles and obtained the same pattern of results. In all three experiments, we also attempted to manipulate the strength of the endowment effect by allowing participants more opportunities in the choice process. Finally, we conclude by discussing our results and their implications.

\section{Experiment 1}

A large body of research supporting ambiguity avoidance has used similar experimental procedures to those adopted by Ellsberg. Typically, in these experiments participants are presented with two urns containing 100 balls. One urn (the unambiguous) contains 50 black and 50 red balls, while the other (the ambiguous) contains unknown quantities of black and red balls. Participants are asked to choose a color and then draw a ball from one of the urns. If they draw their chosen color they win an amount of money, otherwise they win nothing. These experiments have generally shown that for a range of real and hypothetical positive payoffs from $\$ 1$ to $\$ 100$ and when probabilities are not extreme (i.e., close to 0 or 1 ) individuals tend to be ambiguity averse. That is, they show a strong preference for the unambiguous urn regardless of whether they have to choose which urn to draw from or to state a price for the gamble (WTP or WTA). However, research on ambiguity avoidance has not investigated the situations where individuals already own gambles and have the opportunity to exchange them, i.e., the effects of possible status quo bias.

The primary objective of Experiment 1 was to investigate whether such a bias affects preferences between ambiguous and unambiguous gambles in a typical Ellsberg situation. 


\subsection{Hypotheses}

Our main prediction was that the status quo bias would reduce the level of ambiguity aversion typically observed when participants are asked to choose to play gambles from either an ambiguous or unambiguous urn. This led to two specific hypotheses.

\section{Hypothesis 1.1. - "Ellsberg”}

First, participants who are not initially endowed with a gamble will be ambiguity averse by choosing to play the unambiguous over the ambiguous gamble.

\section{Hypothesis 1.2. - "Status quo"}

Second, that the degree of ambiguity aversion will differ between participants who are and are not initially endowed with the ambiguous gamble. Specifically, the former will choose to play (by retaining) the ambiguous gamble to a greater extent than the latter choose the ambiguous option.

We also sought to enhance the degree of endowment by increasing participants' involvement in the choice process (cf., Koehler, Gibbs, \& Hogarth, 1994). To achieve this, some participants initially endowed with the ambiguous gamble were asked to choose in advance the color of the ball before deciding whether to retain their gamble or exchange it for the unambiguous alternative. We reasoned that this manipulation would encourage participants to imagine actually playing the gamble thereby increasing a sense of ownership and, in so doing, enhance the status quo effect. This led to our third hypothesis: 


\section{Hypothesis 1.3. - "Involvement"}

The proportion of participants retaining the ambiguous gamble will be greater among those who choose the color of the ball prior to as opposed to after deciding to keep or reject the ambiguous gamble (Hypothesis 1.3).

\subsection{Design}

There were three conditions all based on Ellsberg's original experiment. All participants were presented with two urns and required to select a ball from one of the urns and to guess its color, red or black. They were asked to imagine that a correct guess was worth 10 pounds (sterling). These were hypothetical payoffs but participants were asked to answer as if they were playing with real money. Participants were presented with information about the numbers of red and black balls in each of the two urns. For one of the urns (Urn U) the information was unambiguous showing that it contained 100 balls, 50 red and 50 black. For the other (Urn A) the information was ambiguous showing that it also contained 100 balls, but without any information indicating the numbers of each color.

In the first condition (Control), a replication of the Ellsberg procedure, participants had to choose whether to gamble using Urn A or Urn U. In the second condition (Status Quo 1) participants were given a ticket to play gamble A based on Urn

A. They were then presented with a new gamble U, based on Urn U, and asked whether they wanted to keep their ticket for gamble A or to exchange it for the right to play gamble U. The third condition (Status Quo 2) was the same as Status Quo 1 in all respects except that participants chose which color they wanted before being given the opportunity to keep or exchange the ticket. Since the primary concern of this and the other experiments in this article was to investigate whether the status quo bias affects 
ambiguity aversion, the ambiguous gamble was always presented first and the unambiguous second when gambles were presented successively.

\subsection{Procedure}

There were three experimental sessions, one for each of the conditions described above. Participants received general instructions about how to complete the task and were shown the two urns. All task relevant information was typed on separate sheets. For the first condition, the gamble information was presented on a single sheet. For the second and third conditions participants were initially presented with the description of Gamble A and a ticket that contained a statement indicating the right to play the gamble. Then they were presented with a new piece of paper describing Gamble $U$ and they had to tick a box to indicate whether they wished to retain Gamble A or exchange it for Gamble U. In the third condition, participants had to choose which color they would gamble on before receiving information about Gamble U. Finally, one ball was selected from each urn in front of the participants, thereby providing them with feedback on their choice.

\subsection{Participants}

180 participants were recruited from the undergraduate population of Leeds University Business School (105 female, 75 male). The average age of participants was 20 years. They attended one of three sessions dedicated to the Control $(\mathrm{N}=72)$, Status Quo $1(\mathrm{~N}=41)$ or Status Quo $2(\mathrm{~N}=67)$ conditions respectively. 


\subsection{Results}

The number of participants choosing to play Gamble A and Gamble U under the three conditions is presented in Table 1 . The first row of data in this table shows that under control conditions most participants preferred Gamble U over Gamble A $(\mathrm{Z}=$ 1.89, $\mathrm{p}<0.05)$. These findings are consistent with the Ellsberg hypothesis (1.1) predicting ambiguity aversion.

Insert Table 1 about here

The second and third rows of data in this table present data that are consistent with Hypothesis 1.2 predicting a status quo bias. In particular, significantly more participants retained Gamble A in the Status Quo Groups than those who chose Gamble A in the Control Group. This was supported by two-sample proportions tests $(Z=-2.05$, $\mathrm{p}<0.05$ Status quo 1 setting; $\mathrm{Z}=-4.57, \mathrm{p}<0.001$ Status quo 2 setting).

Finally, the data are also consistent with Hypothesis 1.3 (Involvement) that predicted a stronger status quo bias when participants are asked which color ball they would choose before deciding whether to keep or exchange Gamble A for Gamble U. The proportion of individuals deciding to keep their ticket for Gamble A in Status Quo Group 2, who chose before deciding, was significantly greater than that in Status Quo Group 1 who did not choose before deciding $(Z=1.72, \mathrm{p}<0.05)$.

\subsection{Discussion}

Support for Hypothesis 1.1 (Ellsberg) is consistent with a large body of previous research showing ambiguity avoidance (e.g. Ellsberg, 1961; Raiffa, 1961; Becker \& Brownson, 1964; Yates \& Zukowsi, 1976; Cohen, Jaffray, \& Said, 1985; Eisenberger \& 
Weber, 1995). The support for Hypothesis 1.2 (Status quo) is important in that, to our knowledge, it is the first time that being endowed with an ambiguous gamble has been shown to reduce ambiguity avoidance. We had predicted that this manipulation would enhance the degree of endowment and thereby support the role of the status quo in moderating ambiguity avoidance. Indeed, this latter finding suggests that the existence of conditions under which ambiguity avoidance may be replaced by ambiguity seeking and that individuals may demand compensation to switch from an ambiguous to an unambiguous gamble.

Support for our prediction of decreased ambiguity avoidance when participants were asked, in advance, which color ball they would choose (Involvement) raises issues concerning the mechanisms underlying this effect. In investigating why people are reluctant to exchange lottery tickets, Bar-Hillel and Neter (1996) argued that regret associated with the possibility of experiencing foregone gains was a major factor. We believe that regret may also play a role in our findings. When participants in our experiment chose a color in advance, they were exposing themselves to more regret than would be implied by simply keeping or rejecting the ambiguous gamble.

Three features of Experiment 1 demand further comment. First, participants were asked to imagine that correctly guessing the color of the ball drawn from the urn was worth $£ 10$. It is important to determine whether these effects would also occur with real pay-offs (Camerer \& Hogarth, 1999). Second, Experiment 1 was based on a between-subjects analysis of participants' preferences for ambiguous and unambiguous gambles. A stronger test of the effects of the status quo bias on ambiguity aversion would involve a within-subjects analysis where participants' preferences for equivalent ambiguous and unambiguous gambles are compared across status quo and control conditions. Third, in Experiment 1 we demonstrated that asking participants to choose 
the color of the ball prior to being offered the opportunity to exchange the gamble decreased ambiguity avoidance. Before attributing this to the status quo bias, it is important to investigate other factors known to increase the bias to see whether they also affect ambiguity avoidance. One such factor, source preference, refers to the finding that the value associated with a good is higher when individuals pre-select it rather than when it is given to them (Loewenstein \& Issacharoff, 1994). The primary purpose of Experiment 2 is to investigate the impact of these three issues on the findings reported in Experiment 1.

\section{Experiment 2}

\subsection{Hypotheses}

The purpose of Experiment 2 was to analyze status quo bias effects on ambiguity avoidance using between- and within-subjects analyses and with incentives. Similar to Experiment 1 participants were presented with ambiguous and unambiguous gambles associated with drawing a ball from an urn filled with colored balls. Participants had to choose between pairs of ambiguous and unambiguous gambles on two occasions, once when they had been previously endowed with the ambiguous gambles and once when there was no prior endowment. Since separate groups of participants completed these activities in one of two orders, it is possible to compare the first responses of these two groups in order to undertake a between-subjects analysis of the status quo bias.

\section{Hypothesis 2.1. - "Status quo between subjects"}

On the basis of the results from Experiment 1, we predicted that the proportion of individuals preferring to retain the right to participate in an ambiguous gamble (when 
offered to exchange it for its unambiguous counterpart) will be greater than the proportion of individuals choosing the ambiguous alternative where there is no prior endowment.

\section{Hypothesis 2.2. - "Status quo within subjects"}

Since participants were presented with equivalent pairs of ambiguous and unambiguous gambles under the Neutral and Endowment Conditions, there are four possible profiles of revealed preferences. Table 2 illustrates these four profiles across the two order conditions. Given that all participants completed both activities, the tendency to choose consistently could dilute the status quo bias. Nevertheless, we predicted that when inconsistency occurred, significantly more preference reversals will be consistent, as opposed to inconsistent, with the status quo bias, i.e., an asymmetry between responses in cells 3 and 4 of Table 2 with cell 3 being greater than cell 4 .

Insert Table 2 about here

In order to evaluate further whether factors known to increase the status quo bias also affect ambiguity avoidance, we induced the bias in two ways. Either participants were given three ambiguous gambles by the experimenter and then had an opportunity to exchange each one for its unambiguous counterparts or they were presented with the three ambiguous gambles, asked to choose one of them and then offered the opportunity to exchange the chosen gamble for its unambiguous counterpart. Reasoning that the act of choice would increase a sense of endowment, we predicted: 


\section{Hypothesis 2.3. - "Source preference"}

More participants will retain the ambiguous gamble if they chose it, than if it is given to them by the experimenter.

\subsection{Design}

Three pairs of unambiguous-ambiguous gambles were developed from those used by Smith, Dickhaut, and Pardo (2002) describing urns containing red, blue and yellow balls. The urn corresponding to the unambiguous gamble contained 90 balls, 30 of each color. The urn corresponding to the ambiguous gamble contained 90 balls; 30 were known to be red, each of the remaining balls was either yellow or blue, though the number of each was unknown. The three pairs of gambles were differentiated in terms of the pay-off structure associated with drawing the red, blue and yellow balls from an urn. The structure for each pair is described in Table 3. While these pay-offs were described in terms of points gained from drawing a particular ball, participants were told in advance that points would be converted into pounds at a fixed rate of 5 pounds sterling per 2000 points. All gambles had an expected value of 5 pounds sterling.

Insert Table 3 about here

There were three different schemes for presenting gambles. Under the neutral (N) scheme each pair of ambiguous/unambiguous gambles was presented to participants and they were asked to choose which gamble they preferred to play in each pair. Under the participant-determined status quo (PDSQ) scheme, participants were presented with the three ambiguous gambles, chose which they preferred to play and then took a ticket for the right to play this gamble. Next, they were given an opportunity to either keep the ticket or exchange it for a ticket to play its unambiguous counterpart (i.e., the one with 
the same outcome structure). Under the experimenter determined status-quo (EDSQ) scheme, the experimenter gave each participant three tickets for the right to play each of the ambiguous gambles. Next, participants were given opportunities to keep or exchange each of these tickets for another to play its unambiguous counterpart. In order to undertake both within- and between-subjects' data analysis and to control for order effects, three different groups of participants were formed, with each completing two of the three gambling schemes across two phases of the experiment. Table 4 outlines which schemes each group completed and the order in which they completed them for the first and second experimental phases.

Insert Table 4 about here

\subsection{Procedure}

Participants were given a set of general instructions and a pack containing the decision problems, one per sheet. There were problem-specific instructions on the top of each sheet. Participants were told to work through the problems in order, writing their responses in the spaces provided and then putting the sheets into a folder. They were not allowed to change their responses once they had placed them in the folder. The decision sheets for each of the conditions are included in the Appendix.

For the PDSQ and EDSQ conditions, tickets indicating the right-to-play each gamble described on that sheet were attached (a separate ticket for each gamble described). Whenever they decided not to play a gamble, participants were instructed to place the corresponding ticket into the folder. Otherwise they left the ticket on the desk in front of them.

The experiment was conducted in three different sessions, one for each of the groups described above. All participants were told at the outset that they would each 
receive 5 pounds sterling for participating and that 10 of them, picked at random, would be paid an extra amount to be determined at the end of the session by resolving the outcomes of the gambles they had chosen.

\subsection{Participants}

A total of 78 students at Leeds University Business School (45 female, 33 male) were recruited from undergraduate and master courses. The average age of the participants was 23 years.

\subsection{Results}

To test Hypothesis 2.1 (Status quo between subjects) we undertook a betweensubjects analysis by comparing the three groups in terms of their choice behavior on the task they undertook in the first phase of the experiment. In particular, we determined the percentage of times participants expressed a preference to play ambiguous gambles in (1) the PDSQ condition, where there was just one decision to be made between their preferred ambiguous gamble and its counterpart, (2) the EDSQ, where there were three decisions between each pair of their endowed ambiguous gamble and its unambiguous counterpart, and (3) the $\mathrm{N}$ condition, where there were also these three decisions but without any prior endowment. These data are presented in Table 5 for participants run under each of the three outcome structures.

Insert Table 5 about here

The fourth column of Table 5 outlines the percentage of participants choosing the ambiguous gamble for each group, collapsed across the three pay-off structures. Analysis of these collapsed data supported Hypothesis 2.1 (Status quo between subjects), predicting an endowment effect, by showing a stronger preference for the 
ambiguous gamble under PDSQ and EDSQ as compared with the $\mathrm{N}$ schemes $(\mathrm{t}=-2.37$, $\mathrm{p}<0.01)$. Furthermore, the percentage of participants choosing to play the ambiguous gamble was significantly greater under the PDSQ as compared with the $\mathrm{N}$ scheme $(\mathrm{t}=\mathrm{-}$ 2.67, $\mathrm{p}<0.005)$. Within pay-off structures, this difference was statistically significant for Structure 2, which was preferred by most $(n=20)$ participants $(Z=-2.78, p<0.005)$. This was not, however, the case under pay-off structures 1 and 3, but here sample sizes for PDSQ were small -2 and 8, respectively (all differences in proportions were, however, in the expected direction). A similar analysis comparing EDSQ and $\mathrm{N}$ gambles failed to find a significant difference for both the aggregate data $(t=-0.79, p$ $>0.10)$ and within schemes although all differences were, once again, in the predicted direction.

To summarize, the data support Hypothesis 2.1 (Status quo between subjects) in the presence of a strong endowment manipulation. It is also worth noting that the level of ambiguity avoidance was relatively low in the $\mathrm{N}$ condition (we return to this in the discussion).

Hypothesis 2.2 (Status quo within subjects), predicting inconsistencies in revealed preferences across the two phases will be largely in the direction predicted by the status quo bias, involved comparing the percentage of each type of inconsistency for each group. These data are presented in Table 6 in terms of the overall percentage of inconsistent responses across the two experimental phases (column three) and the percentage of these responses that were and were not in the direction predicted by the status quo bias (columns one and two). For all three groups, a significantly higher percentage of the inconsistencies were found to lie in the direction predicted by the status quo bias (for all three binomial proportion tests $\mathrm{p}<0.005)^{2}$. That is, participants retained an ambiguous gamble when endowed with it, but chose its unambiguous 
counterpart under neutral conditions. It is, however, important to note that the majority of decisions were taken consistently across the two phases of the experiment.

Insert Table 6 about here

To test Hypothesis 2.3 (Source preference), we undertook a between-subjects analysis to determine whether participants were more likely to retain the ambiguous gamble when they chose it, relative to when it had been given to them by the experimenter. In particular, we compared the percentage of decisions to retain the ambiguous gamble in the first phase of the task across the PDSQ and EDSQ conditions. These data, presented in the second and third rows of Table 5, indicate that the status quo bias was stronger when participants initially choose a gamble rather than when it is given to them. However, while the ambiguous gamble was retained more often in the PDSQ than in the EDSQ condition, this effect was only marginally significant $(t=1.49$, $\mathrm{p}=0.073)$

\subsection{Discussion}

The findings of Experiment 2 were consistent with the between-subjects status quo bias hypothesis (Hypothesis 2.1) and replicated Experiment 1 by showing that the proportion of ambiguity seeking choices was greater for participants who had been endowed - as opposed to not been endowed - with ambiguous gambles. However, this effect was not statistically significant for the EDSQ condition involving a weaker status quo manipulation. The findings were also in line with the within-subjects status quo hypothesis (Hypothesis 2.2) in that observed preference reversals under the EDSQ and PDSQ conditions were predominantly consistent with a status quo bias. Specifically, when participants were inconsistent in choosing between the ambiguous and 
unambiguous gambles across the two phases of the task, the observed inconsistency was consistent with the participants keeping the ambiguous gamble when they had been endowed with it.

The findings only marginally confirmed the source preference hypothesis (Hypothesis 2.3) predicting that there would be a stronger status quo effect when participants were allowed to choose an ambiguous gamble rather than when this was given to them by the experimenter. Further confirmation of these effects would suggest that revealed ambiguity attitudes are also affected by the source of the endowment.

While the findings of Experiment 2 provide further support that the status quo can affect attitudes to ambiguity, several authors have emphasized the need to test the applicability of experimental results based on gambling devices to more realistic settings (for a review see Camerer, 1995). Experiment 3 was designed to replicate Experiment 2 in a financial context, using exactly the same gambles but describing them as investment alternatives.

\section{Experiment 3}

The primary purpose of Experiment 3 was to test the three hypotheses examined in Experiment 2 in a financial context. Since previous research has shown that the status quo bias (e.g. Samuelson \& Zeckhauser, 1988) and ambiguity aversion (reviewed in, e.g., Camerer \& Weber, 1992) occur across a broad range of experimental and everyday contexts, we predicted support for all three hypotheses.

\subsection{Design}

The same three pairs of unambiguous - ambiguous gambles used in Experiment 2 were described in Experiment 3 as investment products. Each product offered 
different rewards to participants depending upon the daily performance of a stock that was to be simulated at the end of the experiment. The price of the stock could go up, down or remain the same and this determined the rewards, in points, that participants would receive. For example rather than being told that "If you draw a red ball you win 3000 points" as per Table 3, participants in Experiment 3 were told "If Stock A remains the same you will earn 3000 points."

The unambiguous products included information about the recent performance of the stock. Participants were told that over the last 90 days, the stock had gone up on 30 occasions, down on 30 occasions and remained the same on 30 occasions. For the ambiguous products, the information indicated that over the last 90 days, the stock had remained the same on 30 occasions but there was no information on the frequency with which the price went up or down for the remaining 60 days. Similar to Experiment 2, there were three different groups of participants as described in Table 4.

\subsection{Procedure}

The procedure was identical to Experiment 2. Participants worked through the pack containing the decision problems and received 5 pounds sterling for participating. At the end of each session 10 participants were selected to play one of their chosen investments and they also received the amount generated by playing out this investment. The experiment was conducted in 3 different sessions, corresponding to the 3 groups $\mathrm{N}$, EDSQ and PDSQ.

\subsection{Participants}

A total of 86 students at Leeds University Business School (47 female, 39 male) were recruited from undergraduate and master courses. The average age of the 
participants was 22 years. The characteristics of these groups are comparable to the students participating in Experiment 2.

\subsection{Results}

We tested the three hypotheses in the same way as described in Experiment 2. Hypothesis 2.1 (Status quo between subjects) was evaluated by undertaking a betweensubjects analysis comparing groups in terms of their choice behavior in the first phase of the experiment. Table 7 shows the percentages of times participants revealed a preference for the ambiguous investment over its unambiguous counterpart for each of the three pay-off structures and summed across all three.

Insert Table 7 about here

These data showed that participants preferred the ambiguous investment more frequently under PDSQ and EDSQ conditions than under the $\mathrm{N}$ condition $(\mathrm{t}=-2.24, \mathrm{p}<$ 0.05). Hypothesis 2.1 (Status quo between subjects) was also confirmed by comparing these percentages for the PDSQ and $\mathrm{N}$ condition $(\mathrm{t}=-2.26, \mathrm{p}<0.05)$. The difference between $\mathrm{N}$ and EDSQ was in the direction predicted but not statistically significant $(\mathrm{t}=$ $-1.09, \mathrm{p}=0.14)$

To test Hypothesis 2.2 (Status quo within subjects), we followed the procedure used in Experiment 2 that involved calculating the number of inconsistencies in preference across the two phases of the experiment, comparing the percentage of these in the direction predicted by status quo effects with those in the opposite direction. These data are presented in Table 8 for each of the three groups separately along with information indicating the overall percentage of all stated preferences that were inconsistent across the two experimental phases. While most decisions were taken 
consistently across the two phases of the experiment, for all three groups, a significantly higher percentage of the inconsistencies were in the direction predicted by the status quo bias (for all three binomial proportion tests $\mathrm{p}<0.01)^{3}$. Thus, the predominant type of inconsistency involved participants retaining an ambiguous gamble when endowed with it, but choosing its unambiguous counterpart under neutral conditions.

Insert Table 8 about here

Hypothesis 2.3 (Source preference) predicted a stronger status quo bias in participants that chose the investment in their endowment, prior to deciding whether to exchange it or not, than in participants who were given the investment by the experimenter. While the data presented in Table 7 suggest that the percentage of decisions to keep the ambiguous investment in the PDSQ condition of group 2 was higher than in the EDSQ condition of group 3, this effect was not statistically significant $(\mathrm{t}=0.94, \mathrm{p}=0.176)$

\subsection{Discussion}

Similar to the two previous experiments, Experiment 3 demonstrated a status quo effect. Endowing participants with an ambiguous alternative increased the likelihood that they would retain it when offered an exchange for its unambiguous counterpart. This effect occurred when comparisons were made both within and across individuals. Importantly, Experiment 3 showed that this effect extends beyond a simple gambling situation to one where participants are choosing between hypothetical investments. There was, however, no support for the source preference hypothesis (Hypothesis 3.3) predicting a stronger status quo effect when participants were allowed to choose an ambiguous gamble rather than being given it by the experimenter. While 
the difference between the EDSQ and PDSQ was in the predicted direction, it was not statistically significant. These findings, along with those reported from the other two experiments, are discussed in greater length in the next section.

\section{General discussion}

Our three experiments have outlined a previously unrecognized condition where people do not avoid ambiguity systematically. We have shown that endowing individuals with an ambiguous alternative can significantly decrease ambiguity avoidance. In all three experiments, there was evidence suggesting that participants were more likely to retain an ambiguous alternative over its unambiguous counterpart when they had previously been endowed with it, in comparison to a neutral situation without prior endowment. This effect occurred both within- and between-subjects, with hypothetical and real incentives, and in experimental situations involving choices between both gambles and investments.

An important feature of Experiments 2 and 3 was evidence showing that the effect of the status quo on ambiguity aversion was less in within-subjects as compared with between-subject analyses. These findings are broadly similar to those reported by LeBoeuf and Shafir (2003) in their studies of risky choice framing where a withinsubject analysis comparing participants' first and second responses showed a reduced framing effect. In explaining their findings LeBoeuf and Shafir (2003) argued that normative principles such as consistency and dominance drive decision making when their appropriateness is recognized and, in doing so, may override other factors, such as framing, in determining choice behavior (see also Fiedler, 1988; Tversky \& Kahneman, 1986). In our Experiments 2 and 3, choice problems were presented sufficiently close together that it is highly likely that they were recognized on the second occasion and 
that consistency provided the basis for choice. As well as raising important issues about the appropriateness of within-subjects studies for investigating human decision making, this explanation of our findings also implies that consistency across situations may be another factor that affects ambiguity aversion.

Different suggestions have been made to explain departures from ambiguity avoidance. Some authors, for example, have shown that ambiguity avoidance varies with the range of outcomes and expected probabilities (Kahn \& Sarin, 1988; Einhorn \& Hogarth, 1986). However, given that we have shown different attitudes to ambiguity between pairs of gambles identical in terms of probabilities and outcomes, this cannot explain our findings. Nonetheless, the dependence of ambiguity avoidance on the probability and outcome domains can help explain the relatively low rates of ambiguity aversion in the $\mathrm{N}$ conditions of Experiments 2 and 3 compared to that of Experiment 1.

Other factors also known to affect attitudes towards ambiguity cannot explain our findings. Consider, for example, the competence hypothesis whereby people exhibit ambiguity seeking when knowledgeable about the source of uncertainty (Fox \& Tversky, 1995; Heath \& Tversky, 1991). In Experiments 1 and 2, we used abstract gambling devices with no possibility of differential competence. In Experiment 3, while the content might have evoked feelings of competence, the context was the same for all conditions. Nor can our findings be explained by "other's evaluation" (Curley, Yates, \& Abrams, 1986) where individuals who know that their choices will be evaluated by others reveal greater ambiguity aversion. In all of the conditions of our experiments, participants were aware that they would be told publicly about the outcomes of all the alternatives. Similarly, we can not interpret our findings in terms of the "comparative ignorance" hypothesis (Fox \& Tversky, 1995) whereby the rates of ambiguity aversion decrease when ambiguous and unambiguous options are evaluated in non-comparative 
settings (see also, Chow \& Sarin, 2001, 2002; Fox \& Weber, 2002). In our experiments, despite the fact that the initial status quo alternatives were described in a noncomparative fashion, decisions between ambiguous and unambiguous options allowed for direct comparison.

There are, however, several psychological phenomena related to the status quo bias that seem to provide a more promising explanation of our findings For instance, the existence of some form of anticipated emotional reactions could have influenced the results of our experiments. It has been shown, that anticipation of feelings is more poignant when they involve potential losses from the status quo (Kahneman \& Tversky, 1982). In the context of decisions regarding lottery tickets, the regret anticipated when giving up a potentially winning lottery ticket (for another lottery ticket) has been shown to be higher than the regret anticipated when retaining a ticket in the status quo and running the risk of not changing it for a winning ticket (Bar-Hillel \& Neter, 1996). We suggest that this could also occur when lottery tickets differ in their degree of uncertainty and that the manipulation in Experiment 1, where individuals chose a color for the winning ball, enhanced this anticipation further. Moreover, the source preference effect of the PDSQ conditions of Experiments 2 and 3 is possibly related to the illusion of control (Langer, 1975) and more weight being associated with choosing as opposed to being given alternatives (Loewenstein \& Issacharoff, 1994; Koehler et al., 1994).

The practical significance of our results can be illustrated in terms of our earlier example comparing the decision between investing in stocks or bonds with the decision to exchange stocks (bonds) in one's possession with bonds (stocks). Results suggest that compared to a choice situation, a tendency to stick to stocks (bonds) will emerge when deciding on the exchange and this will be stronger when individuals have chosen the stocks in their endowment in advance. 
In addition, previous research has suggested that individuals are willing to pay a premium to avoid ambiguity. This includes experiments involving choice and judgment. Indeed, in their extensive review, Camerer and Weber (1992) use the unique term "ambiguity premium" to report the degree of ambiguity aversion implied by results of different types of experiments. Since our results show that attitudes towards ambiguity are significantly affected by the reference point from which alternatives are evaluated (having or not having an ambiguous gamble in the endowment), comparisons between results of experiments implying different frames are problematic. We therefore suggest caution in the use of the concept of "ambiguity premium" and question whether previous experiments have captured what individuals are truly willing to pay to avoid ambiguity.

The endowment effects reported here suggest the need to modify descriptive models of decision making under uncertainty. Several models do consider the distinction between different degrees of uncertainty while allowing for the existence of endowment effects in ambiguous settings (e.g., Cumulative Prospect Theory, Kahneman \& Tversky, 1992). However, they do not account for exchanges between ambiguous and risky alternatives.

Finally, the experiments in the present paper can be extended in several ways. In all three experiments reported in this paper participants were asked to express a preference between equivalent ambiguous and unambiguous gambles. They were not, however, allowed to express indifference. Future research is needed to determine whether the opportunity to express indifference affects the impact of the status quo bias on attitudes to ambiguity. In addition, while we have considered the effects of the status quo bias when individuals are endowed with ambiguous prospects, further experiments might look at other frames of reference and response modes and in turn investigate their 
effects across a broader range of probabilities and outcomes. Of particular interest could be to explore the amounts individuals are willing to pay or require to exchange alternatives subject to different degrees of uncertainty in the line of studies that have looked at the values associated with increases and reductions in risk (cf. Viscusi, Magat, and Huber, 1987). 


\section{Notes}

1. Some experiments have considered selling and buying prices for ambiguous and nonambiguous assets. However, here one of the alternatives being exchanged (i.e., cash) has no uncertainty associated with it (Sarin \& Weber, 1993; Eisenberger \& Weber, 1995).

2. In fact, the binomial test for group 3 is inappropriate because there are 3 observations for each participant. However, of the 16 participants, 7 provided a total of 8 inconsistent judgments. Only 1 of these 8 was inconsistent with the status quo bias.

3. Again, the binomial test for group 3 implies 3 decisions per participant. However, of the 17 participants in group 3, 7 provided a total of 9 inconsistent judgments of which only 2 were inconsistent with the status quo bias (i.e., similar to Experiment 2). 


\section{References}

Bar-Hillel, Maya and Efrat Neter (1996), "Why Are People Reluctant to Exchange Lottery Tickets?", Journal of Personality and Social Psychology, 70, 17-27.

Becker, Selwin W. and Fred O. Brownson (1964), "What Price Ambiguity? Or the Role of Ambiguity in Decision-Making", Journal of Political Economy, 72, 62-73.

Camerer, Colin F. (1995), Individual Decision Making. In: Kagel J.K. and Roth A.E. (eds.) The Handbook of Experimental Economics, Princeton, Princeton University Press, 587-703.

Camerer, Colin F. and Robin M. Hogarth (1999), "The Effects of Financial Incentives in Experiments: A Review and Capital-Labor-Production Framework”, Journal of Risk and Uncertainty, 19, 7-42.

Camerer, Colin F. and Martin Weber (1992), "Recent Developments in Modeling Preferences: Uncertainty and Ambiguity", Journal of Risk and Uncertainty, 5, 325-370.

Chow, Clare C. and Rakesh K. Sarin (2001), "Comparative Ignorance and the Ellsberg Paradox", Journal of Risk and Uncertainty, 22, 129-139.

Chow, Clare C. and Rakesh K. Sarin. (2002), "Known, Unknown and Unknowable Uncertainties", Theory and Decision, 52, 127-138.

Cohen, Michele, Jean-Yves Jaffray and Tanios Said (1985), "Individual Behavior under Risk and under Uncertainty: An Experimental Experiment", Theory and Decision, 50, 360-394.

Curley, Shawn P., Frank Yates and Richard A. Abrams (1986), "Psychological Sources of Ambiguity Avoidance", Organizational Behavior and Human Decision Processes, 38, 230-256. 
Einhorn, Hillel J. and Robin M. Hogarth (1986), ’Decision Making under Ambiguity”, Journal of Business, 59, 225-250.

Eisenberger, Roselies and Martin Weber (1995), "Willingness-to-Pay and WillingnessTo-Accept for Risky and Ambiguous Lotteries", Journal of Risk and Uncertainty, 10, 223-233.

Ellsberg, Daniel (1961), “Risk, Ambiguity and the Savage Axioms”, The Quarterly Journal of Economics, 75, 643-669.

Fiedler, Klaus (1988), "The Dependence of the Conjunction Fallacy on Subtle Linguistic Factors", Psychological Research, 50, 123 - 129.

Fox, Craig R. and Amos Tversky (1995), “Ambiguity Aversion and Comparative Ignorance", Quarterly Journal of Economics, 110, 585-603.

Fox, Craig R. and Martin Weber (2002), “Ambiguity Aversion, Comparative Ignorance and Decision Context", Organizational Behavior and Human Decision Processes, 88, 476-498.

Heath, Chip and Amos Tversky (1991), "Preference and Belief: Ambiguity and Competence in Choice under Uncertainty", Journal of Risk and Uncertainty, 4, $5-28$.

Kahn, Barbara and Rakesh K. Sarin (1988), "Modelling Ambiguity in Decisions under Uncertainty", Journal of Consumer Research, 15, 265-272.

Kahneman, Daniel and Amos Tversky (1979), "Prospect Theory: An Analysis of Decision under Risk”, Econometrica, 47, 263-291.

Kahneman, Daniel and Amos Tversky (1982), “The Psychology of Preference”, Scientific American, 246, 160-291.

Kahneman, Daniel and Amos Tversky (1992), “Advances in Prospect Theory: 
Cumulative Representation of Uncertainty", Journal of Risk and Uncertainty, 5, 297-324.

Kahneman, Daniel, Jack L. Knetsch, and Richard H. Thaler (1991), “Anomalies: The Endowment Effect, Loss Aversion and Status Quo Bias”, Journal of Economic Perspectives, 5, 193-206.

Keren, Gideon and Léonie E. M. Gerritsen (1999), "On the Robustness and Possible Accounts of Ambiguity Aversion", Acta Psychologica, 103, 149-172.

Koehler, Jonathan J., Brian J. Gibbs and Robin M. Hogarth (1994), "Shattering the Illusion of Control: Multi-shot versus Single-shot Gambles", Journal of Behavioral Decision Making, 7, 183-191.

Langer, Ellen J. (1975), “The Illusion of Control”, Journal of Personality and Social Psychology, 32, 311-328.

LeBoeuf, Robyn A. and Eldar Shafir (2003), "Deep Thoughts and Shallow Frames: Effortful Thinking and Susceptibility to Framing Effects", Journal of Behavioral Decision Making, 16, 77-92.

Loewenstein, George and Samuel Issacharoff (1994), "Source Dependence in the Valuation of Objects", Journal of Behavioral Decision Making, 7, 157-68.

Mukerji, Sujoy and Jean-Marc Tallon (2001), “Ambiguity Aversion and Incompleteness of Financial Markets”, Review of Economic Studies, 68, 883-904.

Raiffa, Howard (1961), "Risk, Ambiguity, and the Savage Axioms: Comment.", The Quarterly Journal of Economics, 75, 690-694.

Samuelson, William and Richard Zeckhauser (1988), "Status Quo Bias in Decision Making”, Journal of Risk and Uncertainty, 1, 7-59.

Shafir, Eldar (1993), “Choosing versus Rejecting: Why some Options Are Both Better and Worse than Others", Memory and Cognition, 21, 546-556. 
Shafir, Eldar, Itamar Simonson and Amos Tversky (1993), "Reason-Based Choice”, Cognition, 49, 1-36.

Smith, Kip, John Dickhaut, Kevin McCabe and Jose V. Pardo (2002), "Neuronal Substrates for Coice under Ambiguity, Risk, Gains, and Losses”, Management Science, $48,711-718$.

Thaler, Richard H. (1980), "Toward a Positive Theory of Consumer Choice", Journal of Economic Behavior and Organization, 1, 39-60.

Tversky, Amos and Daniel Kahneman (1986), "Rational Choice and the Framing of Decisions", Journal of Business, 59, s251 - s278.

Viscusi, W. Kip, Wesley A. Magat, and Joel Huber (1987), “An Investigation of the Rationality of Consumer Valuations of Multiple Health Risks". RAND Journal of Economics, 18, 465-479.

Yates, J. Frank and Lisa G. Zukowski (1976), "Characterization of Ambiguity in Decision Making", Behavioral Science, 21, 19-25. 
Table 1. Number of individuals within each of the three groups deciding to play the unambiguous and ambiguous gamble

\begin{tabular}{|c|c|c|c|}
\hline & Unambiguous & Ambiguous & TOTAL \\
\hline Control & 44 & 28 & 72 \\
\hline Status quo 1 & 17 & 24 & 41 \\
\hline Status quo 2 & 17 & 50 & 67 \\
\hline
\end{tabular}


Table 2. The four possible profiles of within-subjects revealed preferences

\begin{tabular}{|c|c|c|c|}
\cline { 3 - 4 } \multicolumn{2}{c|}{} & \multicolumn{2}{c|}{ ENDOWMENT CONDITION } \\
\cline { 3 - 4 } \multicolumn{2}{c|}{} & KEEP AMBIGUOUS & CHANGE TO \\
UNAMBIGUOUS \\
\hline NEUTRAL & AMBIGUOUS & $\begin{array}{c}\text { 1- Consistently ambiguity } \\
\text { seeking }\end{array}$ & $\begin{array}{c}\text { 3- Preference } \\
\text { reversal consistent } \\
\text { with status quo bias }\end{array}$ \\
\cline { 2 - 4 } CONDITION & CHOOSE & $\begin{array}{c}4 \text { - Preference } \\
\text { reversal inconsistent } \\
\text { with status quo bias }\end{array}$ & $\begin{array}{c}\text { 2- Consistently ambiguity } \\
\text { averse }\end{array}$ \\
\hline
\end{tabular}


Table 3. Payoff structures for the three gambles.

\begin{tabular}{|c|c|c|}
\hline Pay-off structure 1 & Pay-off structure 2 & Pay-off structure 3 \\
\hline If you draw a red ball & If you draw a red ball & If you draw a red ball \\
you win 3000 points. & you win 4000 points. & you win 5000 points. \\
If you draw a blue ball & If you draw a blue ball & If you draw a blue ball \\
you win 3000 points. & you win 1500 points. & you win 600 points. \\
If you draw a yellow & If you draw a yellow & If you draw a yellow \\
ball you win nothing. & ball you win 500 points. & ball you win 400 points. \\
\hline
\end{tabular}


Table 4. The order in which the three groups of participants completed the gambling schemes

\begin{tabular}{|lll|}
\hline & GAMBLING SCHEME IN 1 & ST \\
\hline PHASE & GAMBLING SCHEME IN 2 \\
\hline ND PHASE & \\
\hline GROUP 2 & Neutral (N) & Participant-determined status quo (PDSQ) \\
\hline GROUP 3 & Experimenter-determined status quo (EDSQ) & Neutral (N) \\
\hline
\end{tabular}


Table 5. Percentage of decisions to play the ambiguous gamble for each scheme

\begin{tabular}{|c|c|c|c|c|}
\hline$\%$ Ambiguous & Scheme 1 & Scheme 2 & Scheme 3 & Total \\
\hline Neutral (Group 1) & $\begin{array}{c}42 \\
\mathrm{n}=31\end{array}$ & $\begin{array}{c}39 \\
\mathrm{n}=31\end{array}$ & $\begin{array}{c}48 \\
\mathrm{n}=31\end{array}$ & $\begin{array}{c}43 \\
\mathrm{n}=31\end{array}$ \\
\hline $\begin{array}{c}\text { Participant } \\
\text { Determined Status } \\
\text { Quo (Group 2) }\end{array}$ & $\begin{array}{c}67 \\
n=3\end{array}$ & $\begin{array}{c}75 \\
\mathrm{n}=20\end{array}$ & $\begin{array}{c}63 \\
\mathrm{n}=8\end{array}$ & $\begin{array}{c}71 \\
\mathrm{n}=31\end{array}$ \\
\hline $\begin{array}{c}\text { Experimenter } \\
\text { Determined Status } \\
\text { Quo (Group 3) }\end{array}$ & $\begin{array}{c}44 \\
\mathrm{n}=16\end{array}$ & $\begin{array}{c}56 \\
\mathrm{n}=16\end{array}$ & $\begin{array}{c}56 \\
\mathrm{n}=16\end{array}$ & $\begin{array}{c}52 \\
\mathrm{n}=16\end{array}$ \\
\hline
\end{tabular}


Table 6. Percentage of each type of inconsistency and total percentage of inconsistent decisions

\begin{tabular}{|c|c|c|c|}
\cline { 2 - 3 } \multicolumn{1}{c|}{} & \multicolumn{2}{|c|}{ \% Type of inconsistency } & \multirow{1}{*}{} \\
\cline { 2 - 3 } \multicolumn{1}{c|}{} & $\begin{array}{c}\text { Consistent } \\
\text { with Status } \\
\text { Quo Bias }\end{array}$ & $\begin{array}{c}\text { Inconsistent } \\
\text { with Status } \\
\text { Quo Bias }\end{array}$ & \% Inconsistent \\
\hline Group 1 & 73 & 27 & 36 \\
\hline Group 2 & 78 & 22 & 29 \\
\hline Group 3 & 87 & 13 & 17 \\
\hline
\end{tabular}


Table 7. Percentage of decisions to play the ambiguous gamble for each scheme

\begin{tabular}{|c|cccc|}
\hline \% Ambiguous & Scheme 1 & Scheme 2 & Scheme 3 & Total \\
\hline Neutral (Group 1) & 31 & 37 & 37 & 35 \\
& $\mathrm{n}=35$ & $\mathrm{n}=35$ & $\mathrm{n}=35$ & $\mathrm{n}=35$ \\
\hline $\begin{array}{c}\text { Participant } \\
\text { Determined Status } \\
\text { Quo (Group 2) }\end{array}$ & 67 & 58 & 50 & 59 \\
\hline $\begin{array}{c}\text { Experimenter } \\
\text { Determined Status } \\
\text { Quo (Group 3) }\end{array}$ & $\mathrm{n}=6$ & $\mathrm{n}=24$ & $\mathrm{n}=4$ & $\mathrm{n}=34$ \\
\hline
\end{tabular}


Table 8. Percentage of each type of inconsistency and total percentage of inconsistent decisions

\begin{tabular}{|c|c|c|c|}
\cline { 2 - 3 } \multicolumn{1}{c|}{} & \multicolumn{2}{|c|}{ \% Type of inconsistency } & \multirow{1}{*}{} \\
\cline { 2 - 3 } \multicolumn{1}{c|}{} & $\begin{array}{c}\text { Consistent } \\
\text { with Status } \\
\text { Quo Bias }\end{array}$ & $\begin{array}{c}\text { Inconsistent } \\
\text { with Status } \\
\text { Quo Bias }\end{array}$ & \% Inconsistent \\
\hline Group 1 & 91 & 9 & 31 \\
\hline Group 2 & 75 & 25 & 35 \\
\hline Group 3 & 78 & 22 & 18 \\
\hline
\end{tabular}




\section{Appendix. Decision sheets for Experiment 2}

\section{GENERAL INSTRUCTIONS}

Thank you for agreeing to take part in this study. Here are some instructions. Please read them carefully before you proceed.

- The study consists of a set of decision sheets in which you will have to answer according to the particular instructions given.

- Be aware that none of the decision sheets contain the same instructions, although they might seem similar at a first glance. You should therefore, treat each decision afresh.

- You should read one sheet at a time. Every time, you must turn the page, read and follow the instructions. You will not be allowed to go back and change the choices you made. Make sure that you have read the instructions carefully before answering.

- Each person has different options from yours, so please do not discuss your answers with anyone else. There are no right or wrong answers to the questions. This study is interested in your personal preferences.

- At the end of the session each participant will receive a financial reward for participation (5 pounds). An additional payoff will be given to ten participants picked at random. The amount will depend on how many points have been won. Every 2000 points are worth 5 pounds. Point scoring opportunities are described on every decision sheet. 


\section{NEUTRAL CONDITION}

(Each pair was presented and the others of the pairs randomized)

\section{GAME A:}

Imagine a bag filled with 30 red balls and 60 yellow and blue balls. Out of these sixty balls the proportions of yellow and blue balls is unknown.
30 red balls
? blue balls
? yellow balls
90 balls

Game A is to be played with this bag as follows:

If you draw a red ball you win xxx points.

If you draw a blue ball you win xxx points.

If you draw a yellow ball you win xxx points.

\section{GAME B:}

Imagine a bag filled with 30 red balls, 30 yellow balls and 30 blue balls.
30 red balls
30 blue balls
30 yellow balls
90 balls

Game B is to be played with this bag as follows:

If you draw a red ball you win xxx points.

If you draw a blue ball you win xxx points.

If you draw a yellow ball you win xxx points.

You have to choose one of the two games:

TO ANSWER PLEASE TICK ON THE APPROPRIATE BOX

I want to play GAME A

I want to play GAME B 


\section{PARTICIPANT DETERMINED STATUS QUO CONDITION}

(The three gambles were presented in randomized orders)

Please imagine a bag containing 30 red balls and 60 yellow and blue balls. Out of these sixty balls the proportions of yellow and blue balls is unknown.

30 red balls

? blue balls

? yellow balls

90 balls

Three games to be played with this bag are described next. You have to choose one of the games. Attached to this sheet is a ticket that gives you the right to participate in the game you select.

GAME 1 :

If you draw a red ball you win points.

If you draw a blue ball you win 3000 points.

If you draw a yellow ball you win nothing.

\section{GAME 2:}

If you draw a red ball you win 4000 points.

If you draw a blue ball you win 1500 points.

If you draw a yellow ball you win 500 points.

\section{GAME 3:}

If you draw a red ball you win 5000 points.

If you draw a blue ball you win 600 points.

If you draw a yellow ball you win 400 points.

\section{TO ANSWER PLEASE TICK THE APPROPRIATE BOX}

I choose to get a ticket for GAME 1

I choose to get a ticket for GAME 2

I choose to get a ticket for GAME 3 
(After the first decision the following instructions were given corresponding to the chosen gamble $X$ )

You now have in your possession a ticket to play game $\mathrm{X}$. Remember,

The bag corresponding to game $\mathrm{X}$ contains 30 red balls and 60 yellow and blue balls. Out of these sixty balls the proportions of yellow and blue balls is unknown.

30 red balls

? blue balls

? yellow balls

90 balls

Game $\mathrm{X}$ is to be played with this bag as follows:

If you draw a red ball you win xxx points.

If you draw a blue ball you win xxx points.

If you draw a yellow ball you win xxx points.

You now have the option to either keep the ticket or change it for a ticket to play game Y, which is next described:

Imagine a bag which is filled with 30 red balls, 30 blue balls and 30 yellow balls.

$$
\begin{array}{ll}
30 & \text { red balls } \\
30 & \text { blue balls } \\
30 & \text { yellow balls } \\
\hline & 90 \text { balls }
\end{array}
$$

Game $\mathrm{Y}$ is to be played with this bag as follows:

If you draw a red ball you win xxx points.

If you draw a blue ball you win xxx points.

If you draw a yellow ball you win xxx points.

\section{TO ANSWER PLEASE TICK THE APPROPRIATE BOX \\ I want to keep the ticket to play GAME $X$ \\ I want to change the ticket for a ticket to play GAME Y}


EXPERIMENTER DETERMINED STATUS QUO CONDITION

(Each pair was presented and the orders of the pairs randomized)

You have a ticket that gives you the right to play game $\mathrm{X}$, which is to be played as follows.

Imagine a Bag that contains 30 red balls and 60 yellow and blue balls. Out of these sixty balls the proportions of yellow and blue balls is unknown.

30 red balls

? blue balls

? yellow balls

90 balls

Game $\mathrm{X}$ is to be played with this bag as follows:

If you draw a red ball you win xxx points.

If you draw a blue ball you win xxx points.

If you draw a yellow ball you win xxx points.

You now have the option to either keep the ticket or change it for a ticket to play game Y, which is next described:

Imagine a bag which is filled with 30 red balls, 30 blue balls and 30 yellow balls.

30 red balls

30 blue balls

$\underline{30 \text { yellow balls }}$

90 balls

Game $\mathrm{Y}$ is to be played with this bag as follows:

If you draw a red ball you win xxx points.

If you draw a blue ball you win xxx points.

If you draw a yellow ball you win xxx points.

\section{TO ANSWER PLEASE TICK THE APPROPRIATE BOX}

I want to keep the ticket to play GAME X

I want to change the ticket for a ticket to play GAME Y 